\title{
Searching for axion-like particles with proton tagging at the LHC
}

\author{
C. Baldenegro, ${ }^{a}$ S. Fichet, ${ }^{b}$ G. von Gersdorff ${ }^{c}$ and C. Royon ${ }^{a}$ \\ ${ }^{a}$ University of Kansas, \\ Lawrence, Kansas, U.S.A. \\ ${ }^{b}$ ICTP-SAIFR \& IFT-UNESP, \\ R. Dr. Bento Teobaldo Ferraz 271, São Paulo, Brazil \\ ${ }^{c}$ Departamento de Física, Pontifícia Universidade Católica de Rio de Janeiro, \\ Rio de Janeiro, Brazil \\ E-mail: c.baldenegro@cern.ch, sylvain.fichet@lpsc.in2p3.fr, \\ gersdorff@gmail.com, christophe.royon@cern.ch
}

ABSTRACT: The existence of an axion-like particle (ALP) would induce anomalous scattering of light by light. This process can be probed at the Large Hadron Collider in central exclusive production of photon pairs in proton-proton collisions by tagging the surviving protons using forward proton detectors. Using a detailed simulation, we estimate the expected bounds on the ALP-photon coupling for a wide range of masses. We show that the proposed search is competitive and complementary to other collider bounds for masses above $600 \mathrm{GeV}$, especially for resonant ALP production between $600 \mathrm{GeV}$ and $2 \mathrm{TeV}$. Our results are also valid for a $\mathrm{CP}$-even scalar, and the efficiency of the search is independent of the width of the ALP.

Keywords: Phenomenology of Large extra dimensions, Strings and branes phenomenology

ArXiv EPrint: 1803.10835 


\section{Contents}

1 Introduction $\quad 1$

2 The $p p \rightarrow p(\gamma \gamma \rightarrow \gamma \gamma) p$ process

3 Analysis framework $\quad 6$

$\begin{array}{lll}4 & \text { Simulation results } & 7\end{array}$

5 Statistical framework $\quad 8$

6 Results and discussion $\quad 11$

$\begin{array}{llr}7 & \text { Conclusion } & 14\end{array}$

\section{Introduction}

The presence of light (pseudo) scalars coupled to particles of the Standard Model (SM) of particle physics would have numerous consequences from the subatomic to the cosmological scale. These particles might address the longstanding question of why quantum chromodynamics seems to not break the CP symmetry [1], as well as explain a possible component of dark matter. Axion-like particles (ALPs) appear in many extensions of the SM. For example, CP-odd scalars typically appear in the string theory landscape [2-7], or theories with spontaneously broken approximate symmetries [8,9]. A CP-even scalar can for instance be the radion mode from an extra dimension [10], the dilaton arising from spontaneous breaking of conformal symmetry [11], or the radial mode of the symmetrybreaking vacuum in composite Higgs models [12]. Any new neutral spin-0 particle added to the SM typically couples to fermions only via dimension-five operators proportional to the fermion mass, while its dominant coupling to gauge bosons is via dimension-five operators containing derivatives. Therefore, at energies above the top quark mass, these particles are mostly accessible via their couplings to gauge bosons or the Higgs boson. In this paper, we are primarily interested in their coupling to photons.

These particles have been strongly constrained by numerous observations, ${ }^{1}$ some of them by dedicated experiments. A number of these constraints are model-independent, in the sense that they can be shown in the plane of mass versus (pseudo)scalar-photon coupling. This landscape of constraints tends to vanish at high masses, where searches are collider-based. Indeed, searching for a particle relying only on its coupling to photons is not an easy task at a lepton or hadron collider. Bounds obtained from the LEP, Tevatron

\footnotetext{
${ }^{1}$ An up-to-date review can be found in ref. [13].
} 


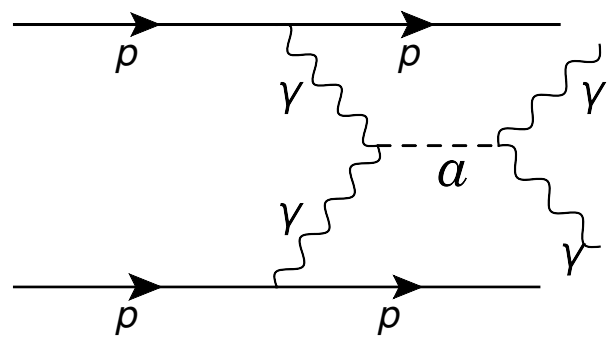

Figure 1. Schematic diagram of an axion-like particle production in two-photon coherent emission in proton-proton collisions. The scattered intact protons are tagged with the forward proton detectors and the photon pair is detected in the central detector.

and LHC in tri-photon, di-photon and mono-photon final states reach the highest masses, but are not very sensitive. Conversely, bounds from on-shell Higgs and $Z^{0}$ boson decays into an ALP in association with visible SM particles have been studied recently in ref. [13] along with their projections for Run-2 of the LHC. The sensitivity found is very good, but the mass reach is necessarily bounded from above by kinematics.

A particularly interesting proposal to study the ALP-photon coupling is via elastic scattering of light-by-light in ultraperipheral heavy-ion collisions, as pointed out in ref. [14]. In these collisions, the coherent photon-photon luminosity is proportional to $Z^{4}(Z=82$ for lead), which enhances significantly the cross section for exclusive diphoton production. Evidence for light-by-light scattering in ultraperipheral heavy-ion collisions was reported by the ATLAS collaboration [15] and the corresponding bounds on the ALP-photon coupling were derived shortly afterwards [16]. These bounds can be quite robust for masses from $1 \mathrm{GeV}$ to $100 \mathrm{GeV}$, with its reach in mass limited from above by the minimum impact parameter in ultraperipheral heavy-ion collisions. However, for larger masses usually accessible in proton-proton ( $\mathrm{p}-\mathrm{p}$ ) collisions at the LHC, the ALP search remains very challenging.

This paper presents an extension of the search for a (pseudo) scalar in light-by-light scattering at the LHC. We propose to search for ALPs in central exclusive diphoton production in p-p collisions (see figure 1),

$$
p p \rightarrow p(\gamma \gamma \rightarrow \gamma \gamma) p
$$

where the photon pair is measured in the central detector and the scattered intact protons are tagged with dedicated forward proton detectors, which are installed symmetrically at a distance of about $210 \mathrm{~m}(220 \mathrm{~m})$ with respect to the interaction points of the CMS (ATLAS) experiment (see figure 2). Using proton tagging, we can reach diphoton invariant masses between $350 \mathrm{GeV}$ and $2 \mathrm{TeV}$, where the acceptance of the forward detectors is nearly $100 \%$ efficient.

The LHC magnets around the interaction points of CMS and ATLAS act as a precise longitudinal momentum spectrometer on the protons that have lost a fraction of their original momentum due to the photon exchange. The forward proton detectors are equipped with charged particle trackers to tag the intact protons. The proton fractional momentum loss $\xi=\Delta p / p$ is reconstructed offline. Compared to other exclusive production searches, which usually rely on vetoes on the detector activity (for example, absence of calorimeter 


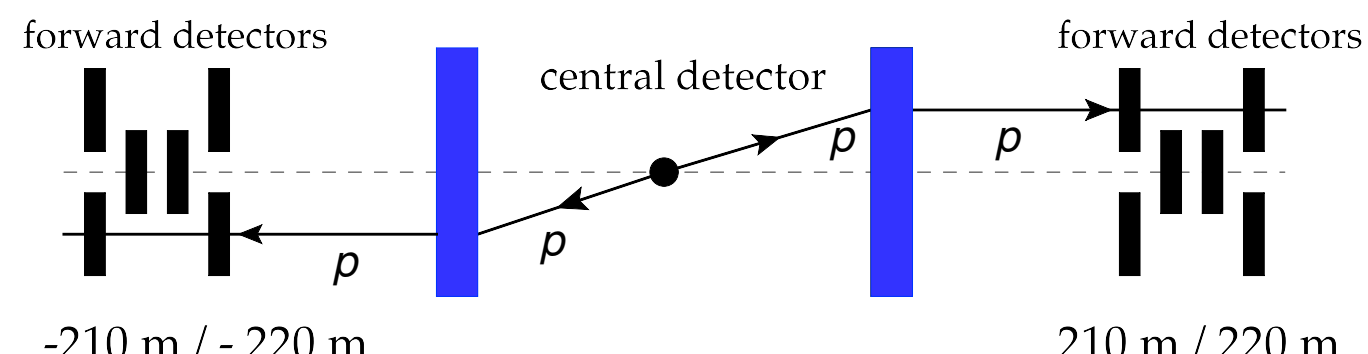

Figure 2. Schematic diagram of the proton tagging method at the LHC in central exclusive processes. The central detector (circle) collects the photon pair. The LHC magnets (blue) act as a precise momentum spectrometer on the outgoing intact protons. The protons pass through the forward detectors (black boxes) and their kinematic information is reconstructed offline. The dashed line represents the beamline.

activity in the forward and backward rapidities above a threshold), the proton tagging method directly measures the proton surviving the coherent photon emission.

The forward detectors, together with the central detector, enable the complete reconstruction of the collision event. This sets a kinematical constraint on the final state that allows an efficient offline selection for central exclusive processes with large background rejection factors. Other studies based on proton tagging at the LHC for new physics searches can be found in [17-38].

The detectors capable of studying central exclusive production in Run-2 of the LHC are the ATLAS Forward Physics (AFP) [39] and the CMS-TOTEM Precision Proton Spectrometer (CT-PPS) [40], which were brought online in 2017 and 2016 respectively. CT-PPS reported the first physics result at the LHC using the proton tagging method by observing the $p p \rightarrow p\left(\gamma \gamma \rightarrow \ell^{+} \ell^{-}\right) p$ reaction [41], which serves as a proof of principle of the proton tagging method to study $\gamma \gamma$ collisions above the electroweak scale. About $40 \mathrm{fb}^{-1}$ of data has been recorded by AFP and CT-PPS for offline analysis at the time of writing this paper.

Qualitatively, the interest of our proposed search method compared to the ones mentioned at the beginning is that the mass reach is limited only by the center-of-mass energy of the proton-proton system and the acceptance of the forward detectors. The photon-photon luminosity for the $\gamma \gamma \rightarrow \gamma \gamma$ subprocess is much smaller than the one in an ultraperipheral heavy-ion collision, but on the other hand the reach in the center-of-mass energy of the photon pair is higher, which enhances the sensitivity for the ALP search since the (pseudo) scalar-photon coupling vertex comes from an effective dimension-5 operator and thus grows rapidly with the diphoton collision energy. Another advantage of the exclusive channel in proton-proton collisions is that it does not need to rely on a dedicated bump search in the diphoton spectrum. Therefore, the results we can obtain are valid for a very broad resonance, which could be missed by an analysis relying on the diphoton mass spectrum lineshape.

The paper is organized as follows. The ALP-induced light-by-light scattering rate is calculated in section 2. Our analysis strategy is then detailed in section 3, and section 4 
summarizes the simulation results. The statistical framework used for the projections is laid down in section 5. Section 6 contains our final results, where we show our projections in the ALP parameter space.

\section{$2 \quad$ The $p p \rightarrow p(\gamma \gamma \rightarrow \gamma \gamma) p$ process}

We compute the production rates for light-by-light scattering in proton-proton collisions using the equivalent photon approximation [42]. In this approximation, the electromagnetic field generated by the fast moving protons can be considered as an intense photon beam. The photons exchanged by the colliding protons are almost on their mass shell (low virtualities $Q^{2}$ ).

The hadronic cross section can be calculated as a convolution of the effective photon fluxes and the $\gamma \gamma \rightarrow \gamma \gamma$ subprocess matrix elements,

$$
\frac{\mathrm{d} \sigma}{\mathrm{d} \Omega}^{p p \rightarrow p(\gamma \gamma \rightarrow \gamma \gamma) p}=\int{\frac{\mathrm{d} \mathcal{L}^{\gamma \gamma}}{\mathrm{d} \hat{s}}}^{\gamma \gamma} \frac{\mathrm{d} \hat{\sigma}}{\mathrm{d} \Omega}{ }^{\gamma \gamma \rightarrow \gamma \gamma} \mathrm{d} \hat{s},
$$

where $\frac{\mathrm{d} \mathcal{L}}{\mathrm{d} \hat{s}}{ }^{\gamma \gamma}$ is the two-photon effective luminosity spectrum, obtained after integrating the photon fluxes over their virtualities ${ }^{2}$ and energies at a fixed two-photon center-of-mass energy $\hat{s}$, and $\frac{\mathrm{d} \hat{\sigma}}{\mathrm{d} \Omega}{ }^{\gamma \gamma \rightarrow \gamma \gamma}$ is the subprocess differential cross section. We use the photon flux computed from the proton elastic electromagnetic form factor [43].

In our treatment we consider a survival probability $\left\langle\mathcal{S}^{2}\right\rangle=0.9$, which quantifies the probability that the protons remain intact after the coherent photon emission. Phenomenology studies suggest that $\left\langle\mathcal{S}^{2}\right\rangle$ can have weak dependence on the invariant diphoton mass $m_{\gamma \gamma}$ and $p_{\mathrm{T}}^{\gamma}$ due to soft rescattering effects between the surviving protons [44, 45], and it might have values down to $\left\langle\mathcal{S}^{2}\right\rangle=0.6$ for masses close to $2 \mathrm{TeV}$ for some photo-produced processes, where the acceptance of the forward detectors vanishes. Ultimately, this nonperturbative quantity has to be measured experimentally by the AFP and CT-PPS experiments. We should mention that in our study we do not take into account polarization effects of the initial-state photons, as is often done in the equivalent photon approximation. Initial-state photons polarizations could yield differences in the production cross section, since they might affect the aforementioned soft rescattering effects [44-46]. To the best of our knowledge, there are no explicit calculations for polarization effects in $p p \rightarrow p(\gamma \gamma \rightarrow \gamma \gamma) p$ scattering, although results for specific processes are known [44, 46]. For purposes of the expected bounds calculation, which will depend on the total yield of the signal rather than on differential features, we will neglect these effects. However, if an ALP is discovered in this channel, a proper treatment of initial-state photons polarizations would be useful to determine the nature of the new particle.

In order to describe the interaction of the (pseudo) scalar $a$ with photons we use the effective interaction models

$$
\mathcal{L}^{+}=\frac{1}{f} a F_{\mu \nu} F^{\mu \nu} \quad(\text { CP-even }), \quad \mathcal{L}^{-}=\frac{1}{f} a F_{\mu \nu} \tilde{F}^{\mu \nu} \quad(\text { CP-odd }),
$$

\footnotetext{
${ }^{2}$ We use the lowest virtuality allowed by kinematics $Q_{\min }^{2}=\frac{2 m_{p}^{2} E_{\gamma}^{2}}{\sqrt{s}\left(\sqrt{s} / 2-E_{\gamma}\right)}$, where $\sqrt{s}$ is the center-of-mass energy of the p-p system. We take the maximum virtuality of $Q_{\max }^{2}=4 \mathrm{GeV}^{2}$.
} 
where $f^{-1}$ is the ALP-photon coupling and $\tilde{F}^{\mu \nu}=\frac{1}{2} \epsilon^{\mu \nu \rho \sigma} F_{\rho \sigma}$. The contributions to the $\gamma \gamma \rightarrow \gamma \gamma$ helicity amplitudes in both cases read

$$
\begin{aligned}
& \mathcal{M}_{++++}=-\frac{4}{f^{2}} \frac{s^{2}}{s-m_{a}^{2}} \\
& \mathcal{M}_{++--}=-(C P) \frac{4}{f^{2}}\left(\frac{s^{2}}{s-m_{a}^{2}}+\frac{t^{2}}{t-m_{a}^{2}}+\frac{u^{2}}{u-m_{a}^{2}}\right), \\
& \mathcal{M}_{+++-}=0,
\end{aligned}
$$

and $\mathcal{M}_{+-+-}(s, t, u)=\mathcal{M}_{++++}(u, t, s), \mathcal{M}_{+--+}(s, t, u)=\mathcal{M}_{++++}(t, s, u)$, where $s, t$ and $u$ are the Mandelstam variables of the diphoton system and $m_{a}$ is the mass of the ALP. This is an effective theory valid roughly up to energy $\sqrt{s} \sim 4 \pi f$. The amplitude grows with $s$ and unitarity would be violated above this scale. The projections we derive in section 6 yield coupling values well below this unitarity bounds. The unpolarized differential crosssection is given by

$$
\frac{d \sigma}{d \Omega}=\frac{1}{128 \pi^{2} s}\left(\left|\mathcal{M}_{++++}\right|^{2}+\left|\mathcal{M}_{+-+-}\right|^{2}+\left|\mathcal{M}_{+--+}\right|^{2}+\left|\mathcal{M}_{++--}\right|^{2}\right) .
$$

Each term in eq. (2.6) in principle contains the SM light-by-light background. However, the production rate for this process is very small within the acceptance of the forward detectors. In particular, the $\mathrm{CP}$ odd and even cases yield the same differential cross section. The scalar being coupled to photons, it has a minimal decay width of

$$
\Gamma(a \rightarrow \gamma \gamma)=\frac{m_{a}^{3}}{4 \pi f^{2}}
$$

In our upcoming projections the decay width of $a$ is a free parameter satisfying $\Gamma \geq$ $\Gamma(a \rightarrow \gamma \gamma)$. The decay width will be parametrized via the branching ratio into photons $\mathcal{B}(a \rightarrow \gamma \gamma)=\Gamma(a \rightarrow \gamma \gamma) / \Gamma$.

It is instructive to examine the amplitudes when taking into account our knowledge of the forward detectors. The forward detectors have access to the process in a given interval of center-of-mass energy $\sqrt{s} \in\left[\sqrt{s}_{0}, \sqrt{s}_{1}\right]$, where $\sqrt{s}_{0}$ is sizeable. For the forward detectors installed at ATLAS and CMS we have roughly $\sqrt{s}_{0} \sim 350 \mathrm{GeV}, \sqrt{s}_{1} \sim 2 \mathrm{TeV}$, where the acceptance is efficient. It follows that one can distinguish three regimes for the diphoton production rate.

If $m_{a}<\sqrt{s}_{0}$, the mass of the particle is negligible from the viewpoint of the detectors, hence the sensitivity will be independent of $m$ and of the width of the particle. Note that the cross section still grows as $s$ in this regime as a consequence of the non-renormalizability of the interaction eq. (2.2), hence the search for a light particle coupled to photons can benefit a lot from an increase in collision energy. If $\sqrt{s}_{0}<m_{a}<\sqrt{s}_{1}$, the scalar is produced resonantly. In that regime a bump search can be performed, unless the resonance is very broad. When the resonance is narrow, the cross section behaves as

$$
\sigma_{\gamma \gamma \rightarrow a \rightarrow \gamma \gamma} \propto f^{-2} \mathcal{B}_{a \rightarrow \gamma \gamma}
$$


Finally if $m_{a}>\sqrt{s}$, the scalar is too heavy to be produced resonantly within the acceptance of the detector. Taking the $m_{a} \gg \sqrt{s}$ limit, the amplitude can then be described by the low-energy effective field theory

$$
\mathcal{L}_{\text {eff }}^{+}=\frac{1}{2 f^{2} m_{a}^{2}}\left(F_{\mu \nu} F^{\mu \nu}\right)^{2}, \quad \mathcal{L}_{\text {eff }}^{-}=\frac{1}{2 f^{2} m_{a}^{2}}\left(F_{\mu \nu} \tilde{F}^{\mu \nu}\right)^{2},
$$

These three regimes will clearly appear on the sensitivity plots.

\section{Analysis framework}

Our event selection treatment follows the method used in refs. [17, 18, 31, 32, 34, 37, 38] and resembles analyses reported by the ATLAS and CMS collaborations on search for exclusive diphoton production in $\mathrm{p}-\mathrm{p}$ and $\mathrm{Pb}-\mathrm{Pb}$ collisions $[15,47]$. We consider proton-proton collisions at a center-of-mass energy of $13 \mathrm{TeV}$ and an integrated luminosity of $300 \mathrm{fb}^{-1}$. We look for photons reconstructed in the barrel region $|\eta|<2.5$, where the reconstruction efficiency is on average $80 \%$ for energetic photons [48, 49]. The photon energy resolution $\Delta E^{\gamma} / E^{\gamma}$ is taken as $1 \%$ since we are dealing with multi-GeV photons. We ask for the leading (subleading) photon to have a minimum transverse momentum of 200 (100) GeV. To better isolate elastically produced photon pairs, we apply a cut on the azimuthal angle separation between the two photons $\left|\Delta \phi^{\gamma \gamma}-\pi\right|<0.01$ and their transverse momentum ratio $p_{\mathrm{T}, 2}^{\gamma} / p_{\mathrm{T}, 1}^{\gamma}>0.95$. We verified the stability of the elastic selection on the signal by varying the azimuthal angle separation between the two photons as $\left|\Delta \phi^{\gamma \gamma}-\pi\right|<0.04$ and $p_{\mathrm{T}, 2}^{\gamma} / p_{\mathrm{T}, 1}^{\gamma}>0.90$. This diphoton selection yields an acceptance of about $80 \%$ on the signal. Finally, we apply a cut on the invariant mass of the photon pair of $600 \mathrm{GeV}$ for background suppression purposes. We assume that the trigger efficiency is close to $100 \%$ at the end of our offline selection.

Since the forward detectors can not get arbitrarily close to the proton beam, and the position of the LHC beam collimators limits the acceptance of the forward detectors from above, the resulting design acceptance on the protons fractional momentum loss is $0.015 \leq \xi \leq 0.15$. We assume that $\xi$ is known to $5 \%$ precision.

The backgrounds for exclusive photon pair production in p-p collisions can be classified in reducible and irreducible backgrounds. The irreducible background comes from the SM light-by-light scattering process, which is induced at one-loop at leading order. This background is greatly reduced within the mass acceptance of the forward proton detectors. Two-gluon exchange between the two colliding protons can lead to a photon pair with intact protons in the final state. However, this background is suppressed more rapidly compared to the SM light-by-light scattering at larger invariant diphoton masses are [31], since we are asking for no additional gluon real emission from the gluon ladder diagram, which formally introduces a Sudakov suppresion factor [50]. Finally, we consider central exclusive prodution of $e^{+} e^{-}$, where the dielectron is misidentified as a photon pair. The misidentification rate electron is about $1 \%$. We find that in our final selection the expected central exclusive production is negligible.

The dominating background is the overlap of a non-exclusive photon pair and uncorrelated protons coming from soft diffractive interactions (see figure 3). Protons originating 

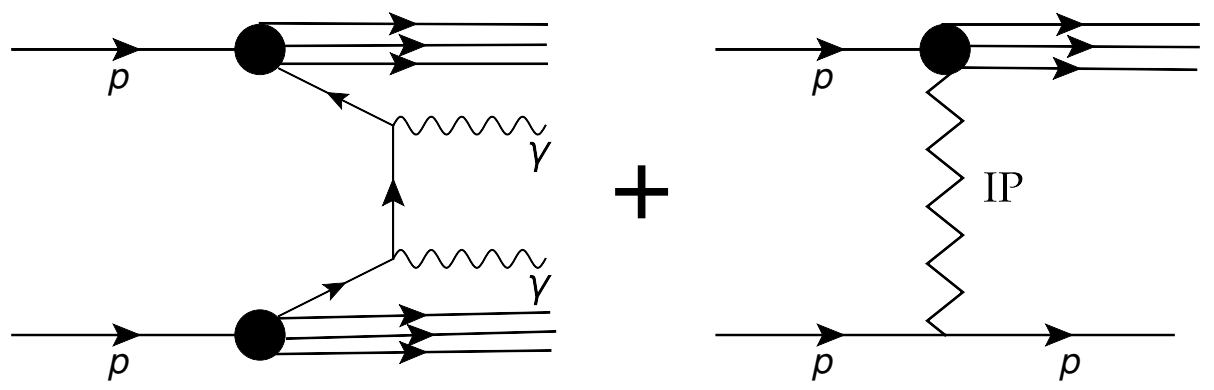

Figure 3. The dominant background for central exclusive diphoton production comes from nonexclusive photon pair production (left) overlapped with uncorrelated protons coming from soft diffractive processes in the additional interactions per bunch crossing (right).

from soft diffractive processes can have fractional momentum losses $\xi$ populating the signal region, and can lead to fake signals. The cross section for diffractive interactions is known to be very large (about $1 \mathrm{mb}$ ), and the number of secondary interactions per bunch crossing (pileup) at the current instantaneous luminosity at the LHC in p-p collisions (50 interactions per bunch crossing at worst) enhances the likelihood of faking the signal. However, central exclusive production events satisfy $m_{\gamma \gamma}=\sqrt{\xi_{1} \xi_{2} s}$ and $y_{\gamma \gamma}=\frac{1}{2} \log \left(\frac{\xi_{1}}{\xi_{2}}\right)$. Thus, we apply a cut $\left|\sqrt{\xi_{1} \xi_{2} s} / m_{\gamma \gamma}-1\right|<0.03$ and $\left|y_{\gamma \gamma}-\frac{1}{2} \log \left(\frac{\xi_{1}}{\xi_{2}}\right)\right|<0.03$. This last point is the key to the precision of this search, as it suppresses a large portion of the background, since non-exclusive photon pairs and protons arising from soft diffractive processes are kinematically uncorrelated.

Other processes contributing to the reducible background are misidentified photon pairs overlapped with soft diffractive protons. We consider non-exclusive $e^{+} e^{-}$pair production (Drell-Yan), since electrons and positrons can fake the diphoton pair, and nonexclusive dijet production, since hard partons can hadronize into a large number of $\pi^{0}$ mesons which subsequently decay into photon pairs, which can fake the photon detection.

Finally, there is a semi-exclusive contribution from diphoton production in double pomeron exchange. This color-singlet exchange can lead to surviving protons and two energetic photons in the final state. However, the production yield falls rapidly as a function of the diphoton invariant mass, and the intact protons and the diphoton are not strongly correlated kinematically since part of the energy is carried by the hadronization of the pomeron. This contribution is negligible in our final selection.

After applying the offline event selection described in this section, we end up with an almost background-free probe for light-by-light scattering in p-p collisions at high diphoton invariant masses, sensitive to cross sections as small as a fraction of a fb, as found before in refs. [31, 37].

\section{Simulation results}

The signal $\gamma \gamma \rightarrow a \rightarrow \gamma \gamma$ subprocess was implemented and generated with the Forward Physics Monte Carlo event generator (FPMC) [51]. FPMC is an event generator for diffractive and photon-induced processes in hadronic collisions. The SM light-by-light scattering 
process is also simulated in FPMC, which includes contributions from charged leptons and the $W$ boson in the one-loop diagram. We also simulated exclusive dielectron production with this generator.

We also employed FPMC for the double pomeron exchange background. FPMC uses the fits based on $\mathrm{H} 1$ inclusive diffractive results [52] of the pomeron in the IngelmanSchlein [53] pomeron parton distribution functions parametrization. The diffractive parton distribution functions are convoluted with the hard scattering processes library in HERWIG 6.5 [54]. FPMC includes a survival probability of 0.03 [50, 55] for pomeron exchange processes. It is not known what the pomeron flux would be at $13 \mathrm{TeV}$, as it has to be constrained experimentally. One may consider this as a theoretical uncertainty. We recomputed the background yield by rescaling it by a factor of 10 and 100 . In neither of these cases we see a significant contamination after the final selection.

Non-exclusive backgrounds, which include diphoton production, dijet production and $e^{+} e^{-}$in Drell-Yan, are simulated in PYTHIA8 [56] using the parton distribution function set NNPDF2.3 QCD+QED LO parametrization. We verified that our results do not depend on the choice of the parton distribution function set. For the misidentified jets, we use the anti- $k_{\mathrm{T}}$ algorithm with the FastJet package [57] with a cone radius $R=0.4$. The probability of tagging at least one proton per diffractive interaction is estimated from the minimum bias library of PYTHIA8 [58]. We assume that the number of secondary interactions per bunch crossing at the interaction points of the LHC follow a Poisson distribution with mean $\mu=50$, the largest number of interactions per bunch crossing at the LHC in Run-2. For each non-exclusive photon pair event, we sample the proton tag probability estimated before for protons coming from soft diffractive events from the additional interactions. If there is at least one proton tagged on each arm, we draw the corresponding $\xi$ value from a parametrization of the differential yield of diffractive protons $\frac{d N}{d \xi} \sim \frac{1}{\xi}$. If more than one proton is present in a given arm, we choose the one that yields the closest mass and rapidity to the diphoton system.

The signal and background yields after the sequential selection cuts described in section 3 can be seen in table 1. After all the selection cuts, we end up with a near backgroundfree probe of light-by-light scattering in p-p collisions. The differential yield for the exclusive diphoton candidates can be seen in figure 4 . The high signal selection efficiency of the exclusive selection is illustrated in figure 5 .

\section{Statistical framework}

For our projections, we need to assume a set of observed data. As commonly done, we assume that no statistical fluctuations are present in these imaginary data, which are usually dubbed "Asimov" data and that we denote here with a prime. As mentioned before, the integrated luminosity is chosen to be $L=300 \mathrm{fb}^{-1}$, which is about the expected integrated luminosity to be taken by CT-PPS and AFP in Run-2.

The observed events follow a Poisson distribution and for this kind of analysis we can safely neglect the systematic uncertainties. The statistics for the events together with the 


\begin{tabular}{|c|c|c|c|c|c|}
\hline Sequential selection & ALP & Excl. SM & DPE $\gamma \gamma$ & $\begin{array}{c}e^{+} e^{-} / \text {dijet } \\
+ \text { pileup }\end{array}$ & $\begin{array}{c}\gamma \gamma \\
+ \text { pile up }\end{array}$ \\
\hline $\begin{array}{c}{\left[0.015<\xi_{1,2}<0.15,\right.} \\
\left.p_{\mathrm{T} 1,(2)}>200,(100) \mathrm{GeV}\right]\end{array}$ & 23.1 & 0.1 & 0.1 & 1.2 & 1246 \\
\hline$m_{\gamma \gamma}>600 \mathrm{GeV}$ & 23.1 & 0.06 & 0 & 0.1 & 440 \\
\hline $\begin{array}{c}{\left[p_{\mathrm{T} 2} / p_{\mathrm{T} 1}>0.95,\right.} \\
\left.\left|\Delta \phi^{\gamma \gamma}-\pi\right|<0.01\right]\end{array}$ & 23.1 & 0.06 & 0 & 0 & 35 \\
\hline$\left|m_{p p} / m_{\gamma \gamma}-1\right|<0.03$ & 21.8 & 0.06 & 0 & 0 & 1.2 \\
\hline$\left|y_{\gamma \gamma}-y_{p p}\right|<0.03$ & 21 & 0.06 & 0 & 0 & 0.2 \\
\hline
\end{tabular}

Table 1. Signal and background yields after applying the event sequential selections. For illustrative purposes, we choose an ALP with mass $m_{a}=1200 \mathrm{GeV}$ and a coupling value of $f^{-1}=0.1 \mathrm{TeV}^{-1}$. We assume an integrated luminosity of $300 \mathrm{fb}^{-1}$ an average of 50 additional interactions per bunch crossing at $\sqrt{s}=13 \mathrm{TeV}$. Excl. stands for the exclusive backgrounds and DPE for double pomeron exchange background. Non-exclusive diphoton overlapped with soft diffractive protons (rightmost column) constitute the dominating background. The first two rows correspond to the diphoton offline preselection. The third row corresponds to the elastic selection. The last two rows correspond to the exclusive selection, with $m_{p p}=\sqrt{\xi_{1} \xi_{2} s}$ and $y_{p p}=\frac{1}{2} \log \left(\frac{\xi_{1}}{\xi_{2}}\right)$.

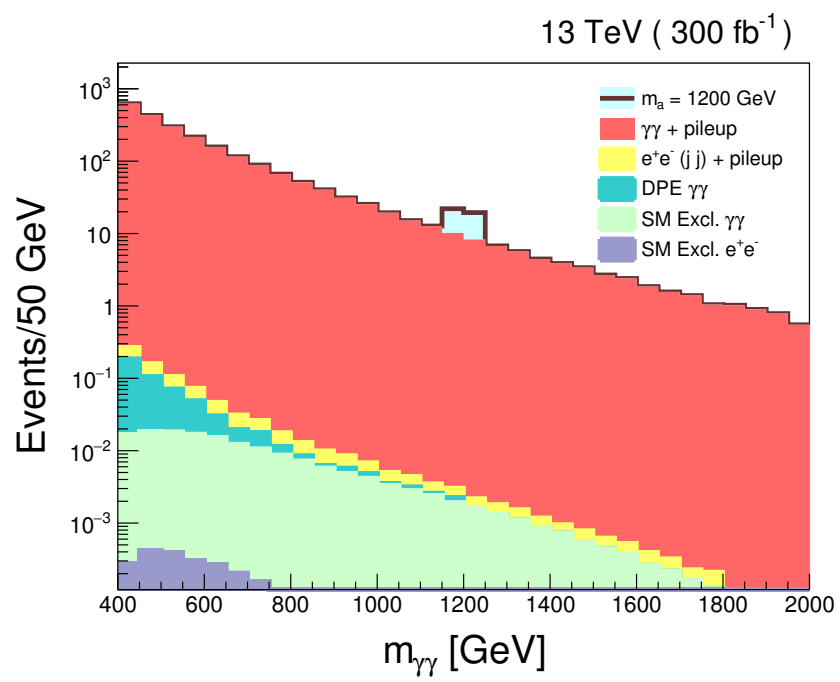

Figure 4. Differential yield as a function of the photon pair invariant mass for exclusive diphoton candidates with two tagged protons within the acceptance $0.015<\xi_{1,2}<0.15$. No elastic or exclusive offline selection is applied for the diphoton candidates in this plot. We assume there are in average 50 secondary interactions per bunch crossing. For illustrative purposes, we show an instance of a resonant ALP production with $m_{a}=1200 \mathrm{GeV}$ and a coupling value $f^{-1}=0.1 \mathrm{TeV}^{-1}$. 

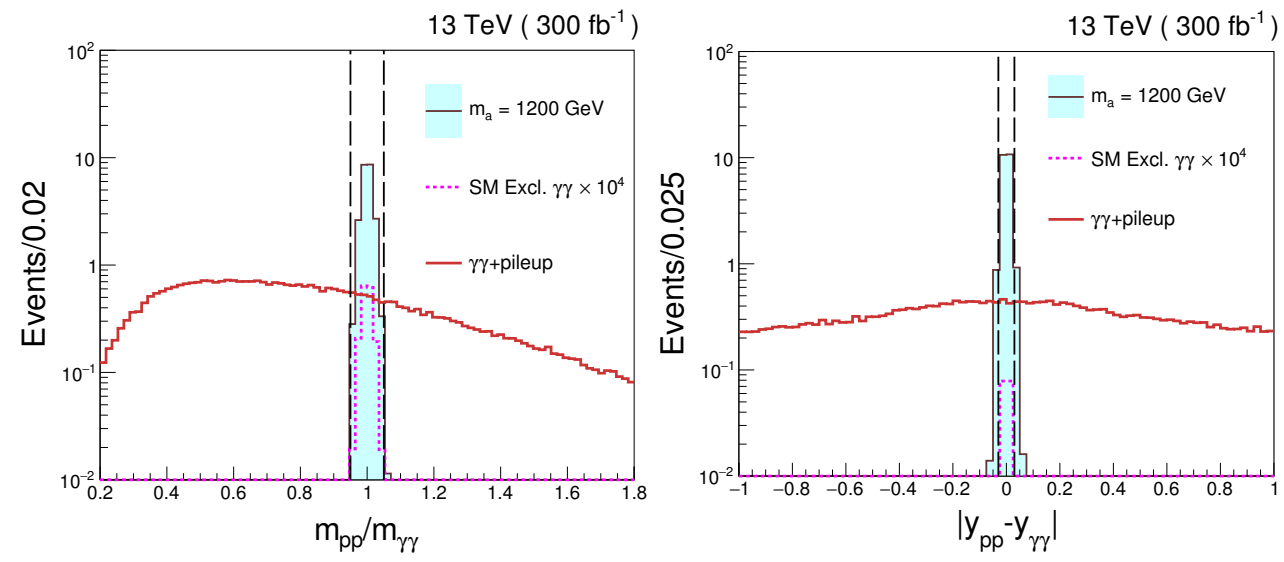

Figure 5. Distributions of the ratio of the diphoton mass reconstructed with the forward detectors $m_{p p}=\sqrt{\xi_{1} \xi_{2} s}$ to the reconstructed diphoton mass $m_{\gamma \gamma}$ (left) and the difference of the diphoton rapidity $y_{\gamma \gamma}$ and the rapidity reconstructed with the forward detectors $y_{p p}=\frac{1}{2} \log \left(\frac{\xi_{1}}{\xi_{2}}\right)$ distribution (right). Diphoton candidates in these plots have passed the elastic selection and the mass lowerbound of $600 \mathrm{GeV}$. A strong correlation between the forward-backward and central information can be seen for the signal (light blue), while for the background (red line) we see these variables are uncorrelated. We select diphoton candidates lying inside the dashed vertical lines. The width of the signal in these plots is caused mainly by the $\xi_{1,2}$ resolution. The integrated luminosity is $300 \mathrm{fb}^{-1}$ and the average number of pileup interactions is $\mu=50$. The intact protons lie within the acceptance $0.015<\xi_{1,2}<0.15$.

prediction for the event rates sets the likelihood function needed for our analysis,

$$
\mathcal{L}(\sigma)=\operatorname{Pr}\left(n^{\prime} \mid b+\sigma L\right), \quad \operatorname{Pr}(\hat{n} \mid n)=\frac{n^{\hat{n}} e^{-n}}{\hat{n} !}
$$

where $b$ is the expected number of events from the background. Thanks to the forward detectors, $b$ is very small, $b \approx 0.2$ for $L=300 \mathrm{fb}^{-1}$.

Our method to obtain the projected $95 \%$ CL exclusion contours is the following. We define the posterior probability density for $\sigma$ as $\mathcal{L}(\sigma) \pi(\sigma)$ where the prior is $\pi(\sigma)=1$ if $\sigma>0$, and 0 otherwise. In order to obtain exclusion contours we will assume that no event is observed, i.e. $n^{\prime}=0$. This is a reasonable scenario because the expected background is $b \approx 0.2$. The non-observation of events sets an upper bound on the signal event rate. The higher posterior density region at $1-\alpha$ credibility level is solved analytically and is simply given by

$$
1-\alpha=\frac{\int_{0}^{\sigma_{\alpha}} L(\sigma) \pi(\sigma)}{\int_{0}^{\infty} L(\sigma) \pi(\sigma)}=1-e^{-\sigma_{\alpha} L}
$$

hence the boundary of the exclusion region is given by

$$
\sigma_{\alpha}=-\frac{1}{L} \log (\alpha) .
$$

Therefore for a $95 \%$ credible interval, one takes $\alpha=0.05$ and the exclusion limit is simply given by $\sigma_{\alpha} \approx 3 L^{-1}$. 
Let us finally comment on the information from signal and background differential distributions. Having resonant production in some regime, it is in general useful to use the characteristic lineshape of a resonant signal to identify it over a background. When doing such "bump search", a likelihood comparing signal and background for every bin has to be used (see e.g. [59]). However, as long as we consider the case $n^{\prime}=0$, there is no need to perform such shape analysis, which would require a precise evaluation of the background on each bin. This is because when $n^{\prime}=0$, the likelihood on each bin is an exponential, hence the likelihood for shape analysis becomes equivalent to the likelihood of eq. (5.1).

\section{Results and discussion}

The expected sensitivity from the exclusive diphoton search can be represented in the $m_{a}-f$ plane. No other assumption is needed except in the region of resonant production where the branching ratio into photons has to be fixed. The expected bound is displayed in figure 6 in the ALP-photon coupling and mass plane for a centrally produced ALP with branching ratio $\mathcal{B}(a \rightarrow \gamma \gamma)=1$. The lowest coupling values range between $0.02 \mathrm{TeV}^{-1}$ and $0.06 \mathrm{TeV}^{-1}$ for masses between $600 \mathrm{GeV}$ to $1.5 \mathrm{TeV}$. The bound increases rapidly from $1.5 \mathrm{TeV}$ to $2 \mathrm{TeV}$ and follows a power-law-like behavior for masses larger than $2 \mathrm{TeV}$ independently of the particle width. For masses below $600 \mathrm{GeV}$, the coupling is independent of the particle width and has a value of about $0.4 \mathrm{TeV}^{-1}$. Bounds with different fixed branching ratios can be seen in figure 7 .

We extracted the existing exclusion limits ${ }^{3}$ from the compilation in ref. [13]. We show a subset of these bounds for masses between $10^{-3} \mathrm{GeV}$ and $2 \mathrm{TeV}$ and coupling values as low as $10^{-3} \mathrm{TeV}^{-1}$, although this landscape of bounds exists down to values of $10^{-15} \mathrm{GeV}$ in mass and $10^{-10} \mathrm{TeV}^{-1}$ in the coupling. Beam dump searches probe resonant production of neutral pseudoscalar mesons in photon interactions with nuclei (Primakoff effect). Different beam dump runs at SLAC collectively yield the area in yellow [60-62]. Upsilon meson decays searched at the CLEO and BaBar experiments [63, 64] exclude the region shaded in green. Bounds from collider-based searches for ALPs include measurements of monophotons with missing transverse energy $\left(e^{+} e^{-} \rightarrow \gamma+\right.$ invisible) at the LEP (orange), triphoton searches on and off the $Z^{0}$ pole $\left(e^{+} e^{-} \rightarrow 3 \gamma\right.$ ) at the LEP (light blue and dark blue), and searches for the same final states in p- $\bar{p}$ collisions at CDF (magenta) and in p-p collisions at the LHC (peach). The derivation of these collider-based bounds are discussed in detail in refs. [14, 65-67]. The region labelled as "Pb-Pb" (light green) was derived in ref. [16] based on the measurement of light-by-light scattering in ultraperipheral heavy-ion collisions by the ATLAS collaboration [15]. These collider-based bounds assume $\mathcal{B}(a \rightarrow$ $\gamma \gamma)=1$. We also include recent constraints presented in ref. [13] based on Higgs boson and $Z$ boson exotic decays $h \rightarrow Z a, h \rightarrow a a$ and $Z \rightarrow \gamma a$, where $a$ decays into a pair of charged leptons or a photon pair, which collectively exclude the parameter space in lavender. These bounds are based on measurements at the LHC in $h \rightarrow Z \gamma, h \rightarrow \gamma \gamma$, $h \rightarrow 4 \gamma$ and $Z \rightarrow 3 \gamma$ final states. Unlike our bounds, these constraints assume a given

\footnotetext{
${ }^{3}$ The ALP-photon coupling in [13] is related to our coupling convention via $f^{-1}=\frac{C_{\gamma \gamma}^{\text {eff }}}{\Lambda} e^{2}$.
} 


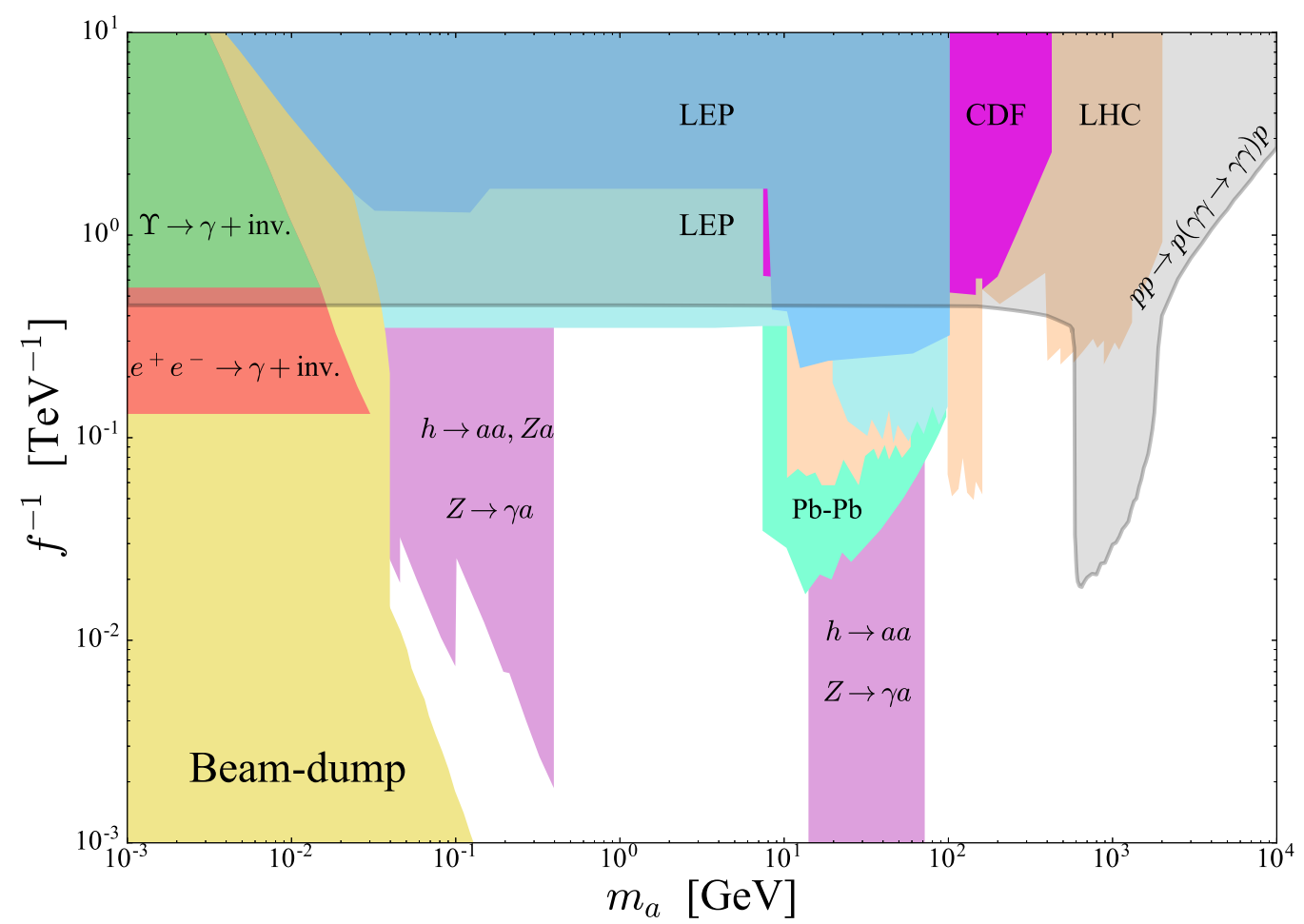

Figure 6. Exclusion regions on the ALP-photon coupling $f^{-1}$ and mass of the ALP $m_{a}$ plane. On light-shaded grey, we have the expected 95\%CL exclusion limit in central exclusive diphoton production events assuming $\mathcal{B}(a \rightarrow \gamma \gamma)=1$ for $300 \mathrm{fb}^{-1}$ in Run- 2 of the LHC. Existing bounds are in solid color, are extracted from ref. [13], and can depend on additional assumptions (see text for details).

coupling value of the ALP to the Higgs boson and $Z$ boson. Another bound (not shown in our figures) was derived based on diphoton resonances searches in the inclusive diphoton cross section measurement at various p-p center-of-mass energies in ref. [68], with the additional assumption of an ALP-gluon coupling.

The LHC region at high mass comes from CMS and ATLAS bump searches in the $\gamma \gamma$ spectrum recasted for ALPs in ref. [65]. A basic extrapolation at $300 \mathrm{fb}^{-1}$ and $\sqrt{s}=13 \mathrm{TeV}$ luminosity tells that this LHC exclusion region would be improved by a factor $\sim 4-5$. In the $0.6-2 \mathrm{TeV}$ mass region, this is still below the expected sensitivity of our exclusive diphoton search by a factor $\sim 3-4$, showing that our method is competitive with respect to standard bump searches. A diphoton bump search as the one in ref. [69] from ATLAS could in principle be competitive with our sensitivity, however in this experimental analysis a very narrow width has been assumed at any mass. This does not correspond to our hypothesis on the width, may not satisfy the theoretical requirement that $\Gamma_{\text {tot }} \geq \Gamma_{\gamma \gamma}$, which may be important for the power of the bump search, hence we have not attempted a comparison with this search.

For $\mathcal{B}(a \rightarrow \gamma \gamma)<1$, the sensitivity of our exclusive diphoton search in the $0.6-2 \mathrm{TeV}$ mass range decreases as shown in figure 7 . This decrease comes simply from the lower signal rate. The total decay width increases for decreasing $\mathcal{B}(a \rightarrow \gamma \gamma)$, (as $\Gamma(a \rightarrow \gamma \gamma)$ is fixed 


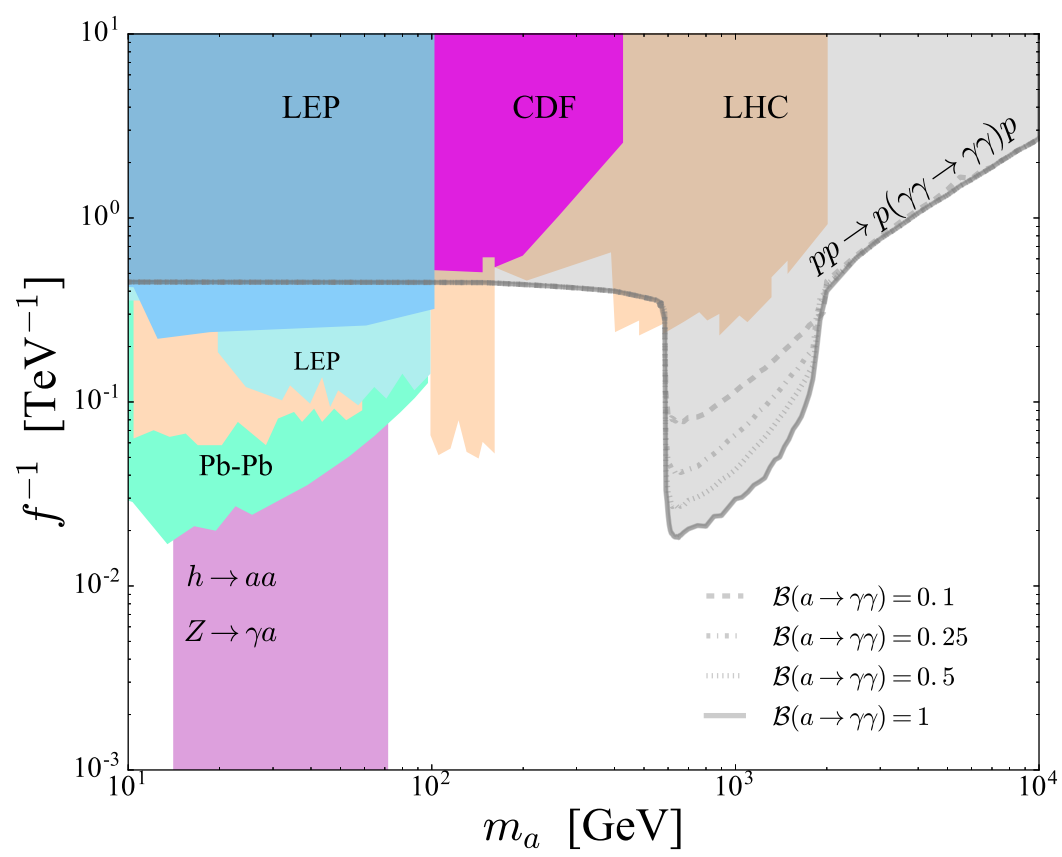

Figure 7. Exclusion regions on the ALP-photon coupling $f^{-1}$ and mass $m_{a}$ plane. On lightshaded grey, we have the expected $95 \%$ CL exclusion limit for $300 \mathrm{fb}^{-1}$ in central exclusive diphoton production events for different branching ratios of the ALP into two photons. Current excluded regions for $\mathcal{B}(a \rightarrow \gamma \gamma)=1$ are in solid color, are extracted from ref. [13], and can depend on additional assumptions (see text for details).

by eq. (2.7)), but the efficiency of our search is width-independent as it consists of a mere counting of the total event number. The region from LHC bump searches shrinks similarly when lowering $\mathcal{B}(a \rightarrow \gamma \gamma)$. However, these searches are valid only for a narrow enough resonance, typically $\Gamma \lesssim 0.05 m_{a}$ [65]. For sufficiently small $\mathcal{B}(a \rightarrow \gamma \gamma)$, the resonance width exceeds this threshold value in the LHC exclusion region at large $m_{a}$, causing the bump search to lose its power. As a consequence our exclusive diphoton search gains extra competitiveness in case of a broad resonance.

For a future collider, a precise prediction would be doable only when knowing enough information on the beam optics and the accelerator's magnetic lattice. This is necessary to assess the feasibility of installing forward proton detectors in such a collider and to determine their acceptance. However, it is worthwhile noticing that if we extrapolate our study to a $100 \mathrm{TeV}$ p-p collider, assuming the same acceptance for the forward detectors as in the LHC, the mass of the ALP would be below the minimum diphoton invariant mass of $1.5 \mathrm{TeV}$, and therefore the sensitivity would be independent of the mass and width of the ALP, and the reach in this region is readily known to be $f \lesssim 6.9 \mathrm{TeV}$. If it is possible to install the forward detectors further away from the interaction point, one could measure smaller proton scattering angles, therefore diphoton invariant masses between $600 \mathrm{GeV}$ and $15 \mathrm{TeV}$ could be reachable. 


\section{Conclusion}

We examined the possibility of searching for axion-like particles in central exclusive production in proton-proton collisions at the center-of-mass energy of $13 \mathrm{TeV}$ for an integrated luminosity of $300 \mathrm{fb}^{-1}$. To quantify the exclusion power, we implemented the helicity amplitudes for light-by-light scattering induced by effective dimension- 5 operators coupling the new (pseudo) scalars to photons in the publicly available Forward Physics Monte Carlo event generator.

To mimic as best as possible the search in AFP or CT-PPS, we made a realistic offline selection on the photon pair as well as on the outgoing intact protons to better isolate quasi-elastic proton-proton collisions. A proper treatment of the background events, with the largest contribution coming from non-exclusive diphoton production overlapped with soft diffractive events, was also presented. We applied a proper statistical treatment to derive the expected bounds on the ALP-photon coupling in the mass reach covered by the exclusive channel, which is the main result of this work.

We have found that the bounds on the ALP-photon coupling for masses above $600 \mathrm{GeV}$ can be improved significantly in the central exclusive photon pair production channel. These regions are constrained by standard LHC bump searches. In the $0.6-2 \mathrm{TeV}$ range we expect that our exclusive diphoton search does better than existing bump searches extrapolated for $300 \mathrm{fb}^{-1}$ and $\sqrt{s}=13 \mathrm{TeV}$ by a factor of $\sim 3-4$ in the ALP-photon coupling. Moreover, the standard bump searches are valid only for narrow enough resonances and lose efficiency for broad resonances, which is not the case of our exclusive diphoton search method.

We conclude that ALP search via light-by-light scattering in central exclusive production in p-p collisions complements other LHC searches and competes with them, especially in the $0.6-2 \mathrm{TeV}$ mass range.

\section{Acknowledgments}

This work has been initiated at the LHC Chapter II: The Run for New Physics workshop held at IIP. SF's work is supported by the São Paulo Research Foundation (FAPESP) under grants \#2011/11973 and \#2014/21477-2. CB thanks the financial support provided by the starting research grant of CR as a Distinguished Foundation Professor.

Open Access. This article is distributed under the terms of the Creative Commons Attribution License (CC-BY 4.0), which permits any use, distribution and reproduction in any medium, provided the original author(s) and source are credited.

\section{References}

[1] R.D. Peccei and H.R. Quinn, CP Conservation in the Presence of Instantons, Phys. Rev. Lett. 38 (1977) 1440 [INSPIRE].

[2] E. Witten, Some Properties of O(32) Superstrings, Phys. Lett. B 149 (1984) 351 [INSPIRE]. 
[3] J.P. Conlon, The QCD axion and moduli stabilisation, JHEP 05 (2006) 078 [hep-th/0602233] [INSPIRE].

[4] P. Svrček and E. Witten, Axions In String Theory, JHEP 06 (2006) 051 [hep-th/0605206] [INSPIRE].

[5] A. Arvanitaki, S. Dimopoulos, S. Dubovsky, N. Kaloper and J. March-Russell, String Axiverse, Phys. Rev. D 81 (2010) 123530 [arXiv:0905.4720] [InSPIRE].

[6] B.S. Acharya, K. Bobkov and P. Kumar, An M-theory Solution to the Strong CP Problem and Constraints on the Axiverse, JHEP 11 (2010) 105 [arXiv:1004.5138] [INSPIRE].

[7] M. Cicoli, M. Goodsell and A. Ringwald, The type IIB string axiverse and its low-energy phenomenology, JHEP 10 (2012) 146 [arXiv:1206.0819] [INSPIRE].

[8] E. Masso and R. Toldra, On a light spinless particle coupled to photons, Phys. Rev. D 52 (1995) 1755 [hep-ph/9503293] [INSPIRE].

[9] B. Bellazzini, A. Mariotti, D. Redigolo, F. Sala and J. Serra, R-axion at colliders, Phys. Rev. Lett. 119 (2017) 141804 [arXiv:1702.02152] [INSPIRE].

[10] C. Csáki, J. Hubisz and S.J. Lee, Radion phenomenology in realistic warped space models, Phys. Rev. D 76 (2007) 125015 [arXiv:0705.3844] [InSPIRE].

[11] W.D. Goldberger, B. Grinstein and W. Skiba, Distinguishing the Higgs boson from the dilaton at the Large Hadron Collider, Phys. Rev. Lett. 100 (2008) 111802 [arXiv:0708.1463] [INSPIRE].

[12] S. Fichet, G. von Gersdorff, E. Pontón and R. Rosenfeld, The Excitation of the Global Symmetry-Breaking Vacuum in Composite Higgs Models, JHEP 09 (2016) 158 [arXiv: 1607.03125] [INSPIRE].

[13] M. Bauer, M. Neubert and A. Thamm, Collider Probes of Axion-Like Particles, JHEP 12 (2017) 044 [arXiv: 1708.00443] [INSPIRE].

[14] S. Knapen, T. Lin, H.K. Lou and T. Melia, Searching for Axionlike Particles with Ultraperipheral Heavy-Ion Collisions, Phys. Rev. Lett. 118 (2017) 171801 [arXiv: 1607.06083] [INSPIRE].

[15] ATLAS collaboration, Evidence for light-by-light scattering in heavy-ion collisions with the ATLAS detector at the LHC, Nature Phys. 13 (2017) 852 [arXiv:1702.01625] [INSPIRE].

[16] S. Knapen, T. Lin, H.K. Lou and T. Melia, LHC limits on axion-like particles from heavy-ion collisions, in Photon 2017: International Conference on the Structure and the Interactions of the Photon and 22th International Workshop on Photon-Photon Collisions and the International Workshop on High Energy Photon Colliders CERN, Geneva, Switzerland, May 22-26, 2017, 2017, arXiv:1709.07110 [INSPIRE].

[17] E. Chapon, C. Royon and O. Kepka, Anomalous quartic $W W$ gamma gamma, $Z$ Z gamma gamma and trilinear $W W$ gamma couplings in two-photon processes at high luminosity at the LHC, Phys. Rev. D 81 (2010) 074003 [arXiv:0912.5161] [InSPIRE].

[18] O. Kepka and C. Royon, Anomalous $W W \gamma$ coupling in photon-induced processes using forward detectors at the LHC, Phys. Rev. D 78 (2008) 073005 [arXiv:0808.0322] [INSPIRE].

[19] I. Sahin and S.C. Inan, Probe of unparticles at the LHC in exclusive two lepton and two photon production via photon-photon fusion, JHEP 09 (2009) 069 [arXiv:0907.3290] [INSPIRE]. 
[20] S. Atag, S.C. Inan and I. Sahin, Extra dimensions in $\gamma \gamma \rightarrow \gamma \gamma$ process at the CERN-LHC, JHEP 09 (2010) 042 [arXiv: 1005.4792] [INSPIRE].

[21] R.S. Gupta, Probing Quartic Neutral Gauge Boson Couplings using diffractive photon fusion at the LHC, Phys. Rev. D 85 (2012) 014006 [arXiv:1111.3354] [INSPIRE].

[22] L.N. Epele, H. Fanchiotti, C.A.G. Canal, V.A. Mitsou and V. Vento, Looking for magnetic monopoles at LHC with diphoton events, Eur. Phys. J. Plus 127 (2012) 60 [arXiv: 1205.6120] [INSPIRE].

[23] P. Lebiedowicz, R. Pasechnik and A. Szczurek, Search for technipions in exclusive production of diphotons with large invariant masses at the LHC, Nucl. Phys. B 881 (2014) 288 [arXiv: 1309.7300] [INSPIRE].

[24] S. Fichet and G. von Gersdorff, Anomalous gauge couplings from composite Higgs and warped extra dimensions, JHEP 03 (2014) 102 [arXiv:1311.6815] [INSPIRE].

[25] S. Fichet, G. von Gersdorff, O. Kepka, B. Lenzi, C. Royon and M. Saimpert, Probing new physics in diphoton production with proton tagging at the Large Hadron Collider, Phys. Rev. D 89 (2014) 114004 [arXiv:1312.5153] [INSPIRE].

[26] H. Sun, Probe anomalous tq $\gamma$ couplings through single top photoproduction at the LHC, Nucl. Phys. B 886 (2014) 691 [arXiv:1402.1817] [InSPIRE].

[27] H. Sun, Large Extra Dimension effects through Light-by-Light Scattering at the CERN LHC, Eur. Phys. J. C 74 (2014) 2977 [arXiv:1406.3897] [InSPIRE].

[28] H. Sun, Dark matter searches in jet plus missing energy events in $\gamma p$ collisions at the CERN LHC, Phys. Rev. D 90 (2014) 035018 [arXiv:1407.5356] [INSPIRE].

[29] I. Şahin et al., Graviton production through photon-quark scattering at the LHC, Phys. Rev. D 91 (2015) 035017 [arXiv:1409.1796] [INSPIRE].

[30] S.C. İnan, Dimension-six anomalous tq $\gamma$ couplings in $\gamma \gamma$ collision at the LHC, Nucl. Phys. B 897 (2015) 289 [arXiv: 1410.3609] [INSPIRE].

[31] S. Fichet, G. von Gersdorff, B. Lenzi, C. Royon and M. Saimpert, Light-by-light scattering with intact protons at the LHC: from Standard Model to New Physics, JHEP 02 (2015) 165 [arXiv: 1411.6629] [INSPIRE].

[32] S. Fichet, Prospects for new physics searches at the LHC in the forward proton mode, Acta Phys. Polon. Supp. 8 (2015) 811 [arXiv:1510.01004] [InSPIRE].

[33] G.-C. Cho, T. Kono, K. Mawatari and K. Yamashita, Search for Kaluza-Klein gravitons in extra dimension models via forward detectors at the LHC, Phys. Rev. D 91 (2015) 115015 [arXiv: 1503.05678] [INSPIRE].

[34] S. Fichet, Shining Light on Polarizable Dark Particles, JHEP 04 (2017) 088 [arXiv: 1609.01762] [INSPIRE].

[35] S. Fichet, G. von Gersdorff and C. Royon, Scattering light by light at $750 \mathrm{GeV}$ at the LHC, Phys. Rev. D 93 (2016) 075031 [arXiv:1512.05751] [INSPIRE].

[36] S. Fichet, G. von Gersdorff and C. Royon, Measuring the Diphoton Coupling of a $750 \mathrm{GeV}$ Resonance, Phys. Rev. Lett. 116 (2016) 231801 [arXiv: 1601.01712] [INSPIRE].

[37] C. Baldenegro, S. Fichet, G. von Gersdorff and C. Royon, Probing the anomalous $\gamma \gamma \gamma Z$ coupling at the LHC with proton tagging, JHEP 06 (2017) 142 [arXiv:1703.10600] [INSPIRE]. 
[38] S. Fichet and C. Baldenegro, Anomalous gauge interactions in photon collisions at the LHC and the FCC, in Photon 2017: International Conference on the Structure and the Interactions of the Photon and 22th International Workshop on Photon-Photon Collisions and the International Workshop on High Energy Photon Colliders CERN, Geneva, Switzerland, May 22-26, 2017, 2017, arXiv:1708.07531 [INSPIRE].

[39] L. Adamczyk et al., Technical Design Report for the ATLAS Forward Proton Detector, CERN-LHCC-2015-009, ATLAS-TDR-024 (2015).

[40] M. Albrow et al., CMS-TOTEM Precision Proton Spectrometer, CERN-LHCC-2014-021 (2014).

[41] CMS and TOTEM collaborations, Observation of proton-tagged, central (semi)exclusive production of high-mass lepton pairs in pp collisions at $13 \mathrm{TeV}$ with the CMS-TOTEM precision proton spectrometer, arXiv:1803.04496 [INSPIRE].

[42] H. Terazawa, Two photon processes for particle production at high-energies, Rev. Mod. Phys. 45 (1973) 615 [INSPIRE].

[43] V.M. Budnev, I.F. Ginzburg, G.V. Meledin and V.G. Serbo, The two photon particle production mechanism. Physical problems. Applications. Equivalent photon approximation, Phys. Rept. 15 (1975) 181 [INSPIRE].

[44] L.A. Harland-Lang, V.A. Khoze and M.G. Ryskin, Exclusive physics at the LHC with SuperChic 2, Eur. Phys. J. C 76 (2016) 9 [arXiv:1508.02718] [InSPIRE].

[45] V.A. Khoze, A.D. Martin and M.G. Ryskin, Multiple interactions and rapidity gap survival, J. Phys. G 45 (2018) 053002 [arXiv:1710.11505] [InSPIRE].

[46] V.A. Khoze, A.D. Martin and M.G. Ryskin, Photon exchange processes at hadron colliders as a probe of the dynamics of diffraction, Eur. Phys. J. C 24 (2002) 459 [hep-ph/0201301] [INSPIRE].

[47] CMS collaboration, Search for exclusive or semi-exclusive photon pair production and observation of exclusive and semi-exclusive electron pair production in pp collisions at $\sqrt{s}=7 \mathrm{TeV}$, JHEP 11 (2012) 080 [arXiv:1209.1666] [INSPIRE].

[48] CMS collaboration, Performance of Photon Reconstruction and Identification with the CMS Detector in Proton-Proton Collisions at $\sqrt{s}=8 \mathrm{TeV}, 2015$ JINST $10 \mathrm{P} 08010$ [arXiv: 1502.02702] [INSPIRE].

[49] ATLAS collaboration, Electron and Photon Reconstruction with the ATLAS Detector, Nucl. Part. Phys. Proc. 273-275 (2016) 2539 [INSPIRE].

[50] V.A. Khoze, A.D. Martin and M.G. Ryskin, Prospects for new physics observations in diffractive processes at the LHC and Tevatron, Eur. Phys. J. C 23 (2002) 311 [hep-ph/0111078] [INSPIRE].

[51] M. Boonekamp et al., FPMC: A generator for forward physics, arXiv:1102.2531 [INSPIRE].

[52] C. Royon, L. Schoeffel, S. Sapeta, R.B. Peschanski and E. Sauvan, A global analysis of inclusive diffractive cross sections at HERA, Nucl. Phys. B 781 (2007) 1 [hep-ph/0609291] [INSPIRE].

[53] G. Ingelman and P.E. Schlein, Jet Structure in High Mass Diffractive Scattering, Phys. Lett. B 152 (1985) 256 [INSPIRE].

[54] G. Corcella et al., HERWIG 6.5 release note, hep-ph/0210213 [INSPIRE]. 
[55] E. Gotsman, E. Levin, U. Maor, E. Naftali and A. Prygarin, Survival probability of large rapidity gaps, in HERA and the LHC: A workshop on the implications of HERA for LHC physics: Proceedings Part A, pp. 221-241, 2005, hep-ph/0511060 [INSPIRE].

[56] T. Sjöstrand, S. Mrenna and P.Z. Skands, A Brief Introduction to PYTHIA 8.1, Comput. Phys. Commun. 178 (2008) 852 [arXiv:0710.3820] [InSPIRE].

[57] M. Cacciari, G.P. Salam and G. Soyez, FastJet User Manual, Eur. Phys. J. C 72 (2012) 1896 [arXiv: 1111.6097] [INSPIRE].

[58] C. Royon, M. Saimpert, O. Kepka and R. Zlebcik, Timing detectors for proton tagging at the LHC, Acta Phys. Polon. B Proceedings Supplement 7 (2014) 735.

[59] F. Ferreira, S. Fichet and V. Sanz, On new physics searches with multidimensional differential shapes, Phys. Lett. B 778 (2018) 35 [arXiv:1702.05106] [INSPIRE].

[60] E.M. Riordan et al., A Search for Short Lived Axions in an Electron Beam Dump Experiment, Phys. Rev. Lett. 59 (1987) 755 [inSPIRE].

[61] J.D. Bjorken et al., Search for Neutral Metastable Penetrating Particles Produced in the SLAC Beam Dump, Phys. Rev. D 38 (1988) 3375 [inSPIRE].

[62] B. Döbrich, J. Jaeckel, F. Kahlhoefer, A. Ringwald and K. Schmidt-Hoberg, ALPtraum: ALP production in proton beam dump experiments, JHEP 02 (2016) 018 [arXiv: 1512.03069] [INSPIRE].

[63] CLEO collaboration, R. Balest et al., $\Upsilon(1 s) \rightarrow \gamma+$ noninteracting particles, Phys. Rev. D 51 (1995) 2053 [INSPIRE].

[64] BABAR collaboration, P. del Amo Sanchez et al., Search for Production of Invisible Final States in Single-Photon Decays of $\Upsilon(1 S)$, Phys. Rev. Lett. 107 (2011) 021804 [arXiv: 1007.4646] [INSPIRE].

[65] J. Jaeckel, M. Jankowiak and M. Spannowsky, LHC probes the hidden sector, Phys. Dark Univ. 2 (2013) 111 [arXiv:1212.3620] [INSPIRE].

[66] K. Mimasu and V. Sanz, ALPs at Colliders, JHEP 06 (2015) 173 [arXiv:1409.4792] [INSPIRE].

[67] J. Jaeckel and M. Spannowsky, Probing MeV to $90 \mathrm{GeV}$ axion-like particles with LEP and LHC, Phys. Lett. B 753 (2016) 482 [arXiv: 1509.00476] [INSPIRE].

[68] A. Mariotti, D. Redigolo, F. Sala and K. Tobioka, New LHC bound on low-mass diphoton resonances, arXiv:1710.01743 [INSPIRE].

[69] ATLAS collaboration, Search for new phenomena in high-mass diphoton final states using $37 \mathrm{fb}^{-1}$ of proton-proton collisions collected at $\sqrt{s}=13 \mathrm{TeV}$ with the ATLAS detector, Phys. Lett. B 775 (2017) 105 [arXiv:1707.04147] [INSPIRE]. 